\title{
ROOT SYSTEMS IN NUMBER FIELDS
}

\author{
VLADIMIR L. POPOV ${ }^{1,2}$ AND YURI G. ZARHIN ${ }^{3}$
}

\begin{abstract}
We classify the types of root systems $R$ in the rings of integers of number fields $K$ such that the Weyl group $W(R)$ lies in the group $\mathcal{L}(K)$ generated by $\operatorname{Aut}(K)$ and multiplications by the elements of $K^{*}$. We also classify the Weyl groups of root systems of rank $n$ which are isomorphic to a subgroup of $\mathcal{L}(K)$ for a number field $K$ of degree $n$ over $\mathbb{Q}$.
\end{abstract}

\section{INTRODUCTION}

In what follows, we call the type of a (not necessarily reduced) root system the type of its Dynkin diagram.

Let $L$ be a free Abelian group of a finite rank $n>0$. We shall consider it as a lattice of full rank in the $n$-dimensional linear space $V:=L \otimes_{\mathbb{Z}} \mathbb{Q}$ over $\mathbb{Q}$. Since every root is an integer linear combination of simple roots, for every type $\mathrm{R}$ of the root systems of rank $n$, there is a subset $R$ in $L$ of rank $n$, which is a root system of type $\mathrm{R}$. However, if the pair $(V, L)$ is endowed with an additional structure, then the Weyl group $W(R)$ of such a realization may be inconsistent with this structure. Say, if the space $V$ is endowed with a scalar product, then it may happen that the group $W(R)$ does not preserve it (for instance, if $n=2$ and $e_{1}, e_{2}$ is an orthonormal basis in $L$, then $\left\{ \pm e_{1}, \pm e_{2}, \pm\left(e_{1}+e_{2}\right)\right\}$ is the root system of type $\mathrm{A}_{2}$ in $V$, whose Weyl group does not consist of orthogonal transformations). Therefore, it is of interest only finding such realizations, the Weyl group of which is consistent with some additional structures on the pair $(V, L)$.

\footnotetext{
1 Steklov Mathematical Institute, Russian Academy of Sciences, Gubkina 8, Moscow 119991, Russia, popovvl@mi-ras.ru

2 National Research University Higher School of Economics, Myasnitskaya 20, Moscow 101000, Russia.

${ }^{3}$ Department of Mathematics, Pennsylvania State University, University Park, PA 16802, USA, zarhin@math.psu.edu

The second named author (Y.Z.) is partially supported by Simons Foundation Collaboration grant \# 585711. Part of this work was done during his stay in MayJuly 2018 at the Max-Planck-Institut für Mathematik (Bonn, Germany), whose hospitality and support are gratefully acknowledged.
} 
A natural source of pairs $(V, L)$ is algebraic number theory, in which they arise in the form $\left(K, \mathscr{O}_{K}\right)$, where $K$ is a number field, and $\mathscr{O}_{K}$ is its ring of integers. In this case, three subgroups are naturally distinguished in the group $\mathrm{GL}_{\mathbb{Q}}(K)$ of nondegenerate linear transformations of the linear space $K$ over $\mathbb{Q}$. The first one is the automorphism group $\operatorname{Aut}(K)$ of the field $K$. The second is the image of the group monomorphism

$$
\text { mult: } K^{*} \hookrightarrow \mathrm{GL}_{\mathbb{Q}}(K)
$$

where $\operatorname{mult}(a)$ is the operator of multiplication by $a \in K^{*}$ :

$$
\operatorname{mult}(a): K \rightarrow K, x \mapsto a x .
$$

The third one is the subgroup $\mathcal{L}(K)$ in $\mathrm{GL}_{\mathbb{Q}}(K)$ generated by $\operatorname{Aut}(K)$ and $\operatorname{mult}\left(K^{*}\right)$.

Definition 1. We say that the type $\mathrm{R}$ of (not necessarily reduced) root systems admits a realization in the number field $K$ if

(a) $[K: \mathbb{Q}]=\operatorname{rk}(\mathrm{R})$;

(b) there is a subset $R$ of $\operatorname{rank} \operatorname{rk}(\mathrm{R})$ in $\mathscr{O}_{K}$, which is a root system of type R;

(c) $W(R)$ is a subgroup of the group $\mathcal{L}(K)$.

In this case, the set $R$ is called a realization of the type $\mathrm{R}$ in the field $K$.

It is worth noting that if we replace $\mathscr{O}_{K}$ by $K$ in (b), we do not obtain a broader concept. Indeed, if $R$ is a subset of $\operatorname{rank} \operatorname{rk}(\mathrm{R})$ in $K$, which is a root system of type $\mathrm{R}$ such that (a) and (c) hold, then there is a positive integer $m$ such that $m \cdot R:=\{m \alpha \mid \alpha \in R\} \subset \mathscr{O}_{K}$. Clearly the set $m \cdot R$ has rank $\operatorname{rk}(\mathrm{R})$, it is a root system of type $\mathrm{R}$, and $W(m \cdot R)=W(R)$.

In view of Definition 1 , if a type $\mathrm{R}$ of root systems admits a realization in a number field $K$, then the group $\mathcal{L}(K)$ contains a subgroup isomorphic to the Weyl group of a root system of type R. Our first main result is the classification of all the cases when the latter property holds:

Theorem 1. The following properties of the Weyl group $W(R)$ of a reduced root system $R$ of type $\mathrm{R}$ and rank $n$ are equivalent:

(i) $W(R)$ is isomorphic to a subgroup $G$ of the group $\mathcal{L}(K)$, where $K$ is a number field of degree $n$ over $\mathbb{Q}$;

(ii) $\mathrm{R}$ is contained in the following list:

$$
A_{1}, A_{2}, B_{2}, G_{2}, 2 A_{1}, 2 A_{1}+A_{2}, A_{2}+B_{2} .
$$

The fact that a subgroup $G$ of the group $\mathcal{L}(K)$ is isomorphic to the Weyl group of a root system of rank $n=[K: \mathbb{Q}]$ and of type $\mathrm{R}$ is not equivalent to the fact that $G=W(R)$, where $R$ is a root system of type $\mathrm{R}$ in $\mathscr{O}_{K}$. This is seen from comparing Theorem 1 with our second 
main result. The latter answers the question of which of the types of root systems in list (3) are realized in number fields:

Theorem 2. For every type $\mathrm{R}$ of root systems (not necessarily reduced), the following properties are equivalent:

(i) there is a number field, in which $\mathrm{R}$ admits a realization;

(ii) $\operatorname{rk}(\mathrm{R})=1$ or 2 .

For $\operatorname{rk}(R)=1$ or 2 , the specific realizations of $R$ in number fields see in Section 2 .

We are grateful to the referee for informative comments, which contributed to making tangible improvements of the text of this paper.

\section{Terminology and notation}

If $R$ is a root system of type $\mathrm{R}$, then the type of the direct sum $m R$ of $m$ copies of $R$ is denoted by $m \mathrm{R}$. We say that $\mathrm{R}$ is irreducible if $R$ is.

All root systems of type $R$ have the same rank denoted by $\operatorname{rk}(R)$.

$\mathrm{A}_{1}^{\prime}$ is the unique type of nonreduced root systems of rank 1 .

By a number field $K$ we mean a field that is an extension of a finite degree of the field $\mathbb{Q}$.

$\mu_{K}$ is the multiplicative group of all roots of unity in $K$; it is a finite cyclic group.

$\mathscr{O}_{K}$ is the ring of all integers in $K$.

$\mathscr{O}_{K}(d)$ is the set of all elements of $\mathscr{O}_{K}$, whose norm is $d$.

$\operatorname{ord}(g)$ is the order of an element $g$ of a group

$\langle g\rangle$ is a cyclic group with the generating element $g$.

$\exp (G)$ is the exponent of a finite group $G$ (i.e., the least common multiple of the orders of all elements of $G$ ).

$[G, G]$ is the commutator subgroup of a group $G$.

$\mathrm{Sym}_{n}$ and $\mathrm{Alt}_{n}$ are respectively the symmetric and alternating groups of permutations of $\{1, \ldots, n\}$.

For a prime number $p$ and a non-zero integer $n$, the $p$-adic valuation of $n$ is denoted by $\nu_{p}(n)$ (i.e., $\nu_{p}(n)$ is the highest exponent $e$ such that $p^{e}$ divides $n$ ).

$\varphi$ is Euler's totient function, i.e., for every integer $d>0$, the value $\varphi(d)$ is the number of positive integers relatively prime to $d$ and $\leqslant d$.

\section{RANKS 1 AND 2}

The following examples show that every type $\mathrm{R}$ of root systems of rank 1 or 2 admits a realization in an number field $K$. 
Root systems of types $\mathrm{A}_{1}$ and $\mathrm{A}_{1}^{\prime}$.

In this case, $K=\mathbb{Q}, \mathscr{O}_{K}=\mathbb{Z}$ and $\mathcal{L}(K)=\operatorname{mult}\left(\mathbb{Q}^{*}\right)$. If $\alpha \in \mathbb{Z}, \alpha \neq 0$, then $R:=\{ \pm \alpha\}$ (respectively, $R:=\{ \pm \alpha, \pm 2 \alpha\}$ ) is a realization of type $\mathrm{A}_{1}$ (respectively, $\mathrm{A}_{1}^{\prime}$ ) in the field $K$, because $W(R)=\langle\operatorname{mult}(-1)\rangle \subset$ $\mathcal{L}(K)$.

Root systems of types $\mathrm{A}_{2}$ and $\mathrm{G}_{2}$.

Let $K$ be the third cyclotomic field: $K=\mathbb{Q}(\sqrt{-3})$. Then $\mathscr{O}_{K}=$ $\mathbb{Z}+\mathbb{Z} \omega$, where $\omega=(1+i \sqrt{3}) / 2$, and $\operatorname{Aut}(K)=\langle c\rangle$, where $c$ is the complex conjugation $a \mapsto \bar{a}$. The bilinear form

$$
K \times K \rightarrow \mathbb{Q},(a, b) \mapsto \operatorname{trace}_{K / \mathbb{Q}}(a \bar{b})=2 \operatorname{Re}(a \bar{b}),
$$

defines on $K$ a structure of Euclidean space over $\mathbb{Q}$. Any element of $\mathcal{L}(K)$, whose order is finite (in particular, any reflection), is an orthogonal (with respect to this structure) transformation.

Since $r_{1}:=\operatorname{mult}(-1) c \in \mathcal{L}(K)$ is a reflection with respect to 1 , the transformation $\rho r_{1} \rho^{-1}$ for every $\rho \in \mathrm{GL}_{\mathbb{Q}}(K)$ is a reflection with respect to $\rho(1)$. For $\rho=\operatorname{mult}(a)$, where $a \in K^{*}$, this yields the element

$$
r_{a}:=\operatorname{mult}\left(-a \bar{a}^{-1}\right) c
$$

of $\mathcal{L}(K)$, which is a reflection with respect to $a$.

The multiplicative group $\left\{ \pm 1, \pm \omega, \pm \omega^{2}\right\}$ of all 6 th roots of 1 coincides with $\mathscr{O}_{K}(1)$. Hence

$$
\mathscr{O}_{K}(1)=\left\{ \pm \alpha_{1}, \pm \alpha_{2}, \pm\left(\alpha_{1}+\alpha_{2}\right)\right\} \text {, where } \alpha_{1}=1, \alpha_{2}=\omega^{2}
$$

Therefore, $\mathscr{O}_{K}(1)$ is the root system of type $\mathrm{A}_{2}$ with the base $\alpha_{1}, \alpha_{2}$. If $a \in \mathscr{O}_{K}(1)$, then $-a \bar{a}^{-1}$ is a root of 1 , hence (5) implies that $r_{a}\left(\mathscr{O}_{K}(d)\right)=$ $\mathscr{O}_{K}(d)$ for any $d$. Therefore, $r_{a} \in W\left(\mathscr{O}_{K}(1)\right)$. Hence $W\left(\mathscr{O}_{K}(1)\right) \subset \mathcal{L}(K)$. This means that $\mathscr{O}_{K}(1)$ is the realization of type $\mathrm{A}_{2}$ in the field $K$.

Since we have

$$
\mathscr{O}_{K}(3)=(1+\omega) \mathscr{O}_{K}(1)
$$

the set $\mathscr{O}_{K}(3)$ is the root system of type $A_{2}$ with the base

$$
\beta_{1}=(1+\omega) \alpha_{1}, \beta_{2}=(1+\omega) \alpha_{2} .
$$

If $a \in \mathscr{O}_{K}(3)$, then (6) implies that $-a \bar{a}^{-1}$ is a root of 1 . This and (5) yield $r_{a}\left(\mathscr{O}_{K}(d)\right)=\mathscr{O}_{K}(d)$ for any $d$. Therefore, $W\left(\mathscr{O}_{K}(3)\right) \subset \mathcal{L}(K)$. Hence $\mathscr{O}_{K}(3)$ is yet another realization of type $\mathrm{A}_{2}$ in the field $K$. 


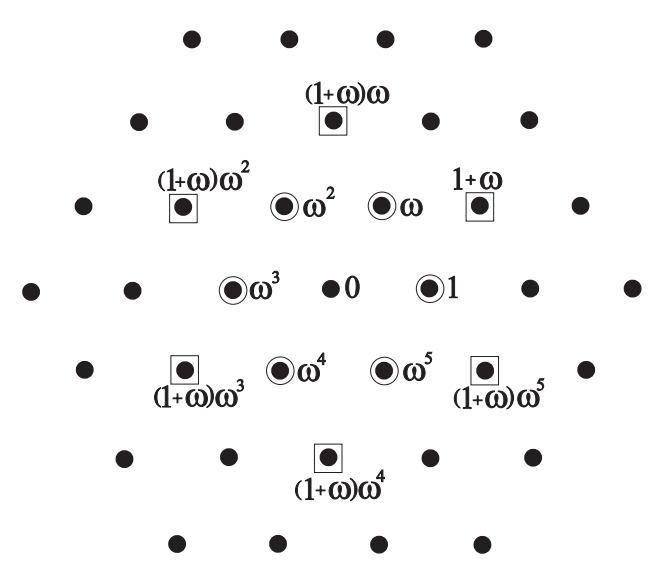

FiruRE 1. Elements of $\mathscr{O}_{K}, \mathscr{O}_{K}(1)$, and $\mathscr{O}_{K}(3)$ are depicted respectively by $\bullet, \bullet$, and $\bullet$

Since we have

$$
\begin{aligned}
\mathscr{O}_{K} & (1) \cup \mathscr{O}_{K}(3) \\
& =\left\{ \pm \alpha_{1}, \pm \beta_{2}, \pm\left(\alpha_{1}+\beta_{2}\right), \pm\left(2 \alpha_{1}+\beta_{2}\right), \pm\left(3 \alpha_{1}+\beta_{2}\right), \pm\left(3 \alpha_{1}+2 \beta_{2}\right)\right\},
\end{aligned}
$$

the set $\mathscr{O}_{K}(1) \cup \mathscr{O}_{K}(3)$ is the root system of type $\mathrm{G}_{2}$ with the base $\alpha_{1}, \beta_{2}$ (this is noted in [6, V, 16]). If $a \in \mathscr{O}_{K}(3), b \in \mathscr{O}_{K}(1)$, then $r_{a}\left(\mathscr{O}_{K}(1)\right)=$ $\mathscr{O}_{K}(1), r_{b}\left(\mathscr{O}_{K}(3)\right)=\mathscr{O}_{K}(3)$. Therefore, $W\left(\mathscr{O}_{K}(1) \cup \mathscr{O}_{K}(3)\right) \subset \mathcal{L}(K)$. Hence $\mathscr{O}_{K}(1) \cup \mathscr{O}_{K}(3)$ is the realization of type $\mathrm{G}_{2}$ in the field $K$.

Root systems $\mathrm{B}_{2}, 2 \mathrm{~A}_{1}, \mathrm{BC}_{2}, 2 \mathrm{~A}_{1}, 2 \mathrm{~A}_{1}^{\prime}$, and $\mathrm{A}_{1}+\mathrm{A}_{1}^{\prime}$.

Let $K$ be the fourth cyclotomic field: $K=\mathbb{Q}(\sqrt{-1})$. Then $\mathscr{O}_{K}=$ $\mathbb{Z}+\mathbb{Z} i$ and $\operatorname{Aut}(K)=\langle c\rangle$, where $c$ is the complex conjugation $a \mapsto \bar{a}$. As above, (4) defines on $K$ a structure of Euclidean space over $\mathbb{Q}$, and any element of $\mathcal{L}(K)$ of finite order (in particular, any reflection) is an orthogonal (with respect to this structure) transformation.

As above, for every $a \in K^{*}$, the element $r_{a} \in \mathcal{L}(K)$, given by formula (5), is a reflection with respect to $a$.

The multiplicative group $\{ \pm 1, \pm i\}$ of all 4 th roots of 1 coincides with $\mathscr{O}_{K}(1)$. Therefore,

$$
\mathscr{O}_{K}(1)=\left\{ \pm \alpha_{1}, \pm \alpha_{2}\right\}
$$

is the root system of type $2 \mathrm{~A}_{1}$ with the base $\alpha_{1}=1, \alpha_{2}=i$. If $a \in \mathscr{O}_{K}(1)$, then $-a \bar{a}^{-1}$ is a root of 1 , hence (5) implies that $r_{a}\left(\mathscr{O}_{K}(d)\right)=\mathscr{O}_{K}(d)$ for any $d$. Therefore, $r_{a} \in W\left(\mathscr{O}_{K}(1)\right)$; whence $W\left(\mathscr{O}_{K}(1)\right) \subset \mathcal{L}(K)$. So, $\mathscr{O}_{K}(1)$ is the realization of type $2 \mathrm{~A}_{1}$ in $K$.

Since we have

$$
\mathscr{O}_{K}(2)=(1+i) \mathscr{O}_{K}(1)
$$


the set $\mathscr{O}_{K}(2)$ is the root system of type $2 \mathrm{~A}_{1}$ with the base

$$
\beta_{1}=(1+i) \alpha_{1}, \beta_{2}=(1+i) \alpha_{2} .
$$

If $a \in \mathscr{O}_{K}(2)$, then (7) implies that $-a \bar{a}^{-1}$ is a root of 1 . This and (15) yield $r_{a}\left(\mathscr{O}_{K}(d)\right)=\mathscr{O}_{K}(d)$ for any $d$. Therefore, $W\left(\mathscr{O}_{K}(2)\right) \subset \mathcal{L}(K)$. Hence $\mathscr{O}_{K}(2)$ is yet another realization of type $2 \mathrm{~A}_{1}$ in $K$.

Since we have

$$
\mathscr{O}_{K}(1) \bigcup \mathscr{O}_{K}(2)=\left\{ \pm \alpha_{1}, \pm \beta_{2}, \pm\left(\alpha_{1}+\beta_{2}\right), \pm\left(2 \alpha_{1}+\beta_{2}\right)\right\}
$$

the set $\mathscr{O}_{K}(1) \cup \mathscr{O}_{K}(2)$ is the root system of type $B_{2}$ with the base $\alpha_{1}, \beta_{2}$. If $a \in \mathscr{O}_{K}(2), b \in \mathscr{O}_{K}(1)$, then $r_{a}\left(\mathscr{O}_{K}(1)\right)=\mathscr{O}_{K}(1), r_{b}\left(\mathscr{O}_{K}(2)\right)=$ $\mathscr{O}_{K}(2)$. Therefore, $W\left(\mathscr{O}_{K}(1) \cup \mathscr{O}_{K}(2)\right) \subset \mathcal{L}(K)$, hence $\mathscr{O}_{K}(1) \cup \mathscr{O}_{K}(2)$ is the realization of type $\mathrm{B}_{2}$ in the field $K$.

Since we have

$$
\mathscr{O}_{K}(4)=2 \mathscr{O}_{K}(1)
$$

the group $W\left(\mathscr{O}_{K}(4)\right)$ coincides with the group $W\left(\mathscr{O}_{K}(1)\right)$. Therefore, $\mathscr{O}_{K}(4)$ is yet another realization of type $2 \mathrm{~A}_{1}$ in $K$. Since

$$
\begin{aligned}
\mathscr{O}_{K}(1) \bigcup \mathscr{O}_{K}(2) \bigcup \mathscr{O}_{K}(4) \\
=\left\{ \pm \alpha_{1}, \pm 2 \alpha_{1}, \pm \beta_{2}, \pm\left(\alpha_{1}+\beta_{2}\right), \pm 2\left(\alpha_{1}+\beta_{2}\right), \pm\left(2 \alpha_{1}+\beta_{2}\right)\right\},
\end{aligned}
$$

the set $\mathscr{O}_{K}(1) \cup \mathscr{O}_{K}(2) \cup \mathscr{O}_{K}(4)$ is the root system of type $\mathrm{BC}_{2}$ with the base $\alpha_{1}, \beta_{2}$. In view of $W\left(\mathscr{O}_{K}(1) \cup \mathscr{O}_{K}(2) \cup \mathscr{O}_{K}(4)\right) \subset \mathcal{L}(K)$, it is the realization of type $\mathrm{BC}_{2}$ in $K$.

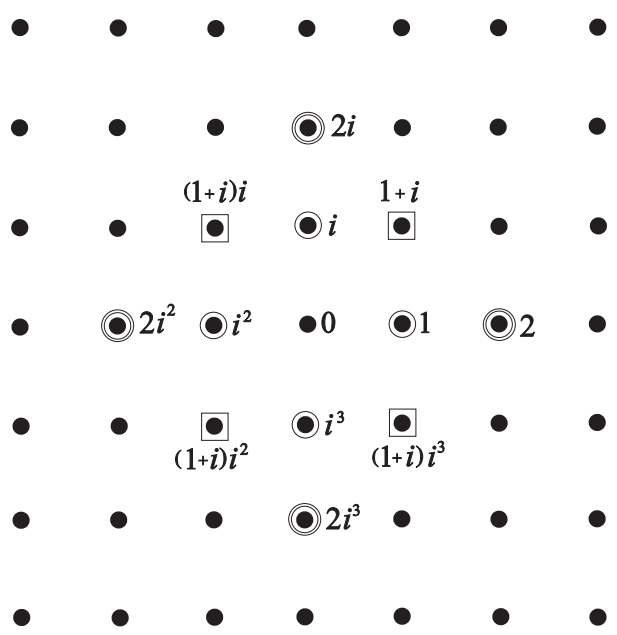

FiRURE 2. Elements of $\mathscr{O}_{K}, \mathscr{O}_{K}(1), \mathscr{O}_{K}(2)$, and $\mathscr{O}_{K}(4)$ are depicted respectively by $\bullet, \bullet, \bullet$, and $(0$

Finally, the realizations of types $2 \mathrm{~A}_{1}^{\prime}$ and $\mathrm{A}_{1} \dot{+} \mathrm{A}_{1}^{\prime}$ in $K$ are respectively

$$
\mathscr{O}_{K}(1) \bigcup \mathscr{O}_{K}(4) \text { and } \mathscr{O}_{K}(1) \bigcup\{ \pm 2\}
$$


Summing up, we have the following

Proposition 1. Every type of root systems of rank $\leqslant 2$ admits a realization in a number field.

\section{Group $\mathcal{L}(K)$ and its finite subGroups}

Below $K$ is a number field of degree $n$ over $\mathbb{Q}$. Given $g \in \mathcal{L}(K)$, we denote

$$
K^{g}:=\{a \in K \mid g(a)=a\} .
$$

If $g \in \operatorname{Aut}(K)$, then $K^{g}$ is a subfield of $K$ and $K / K^{g}$ is a degree ord $(g)$ Galois extension with the Galois group $\langle g\rangle$.

\section{Lemma 1.}

(a) The group $\mathcal{L}(K)$ is a semidirect product of its normal subgroup $\operatorname{mult}\left(K^{*}\right)$ and the subgroup $\operatorname{Aut}(K)$. Therewith,

$$
g \operatorname{mult}(a) g^{-1}=\operatorname{mult}(g(a)) \quad \text { for any } a \in K^{*}, g \in \operatorname{Aut}(K) .
$$

(b) Let $g \in \operatorname{Aut}(K)$ and let

$$
\operatorname{Norm}_{K / K^{g}}: K \rightarrow K^{g}
$$

be the norm map corresponding to the field extension $K / K^{g}$. Then for any $a \in K^{*}$, the following properties are equivalent:

$\left(\mathrm{b}_{1}\right)$ the element mult $(a) g \in \mathcal{L}(K)$ has finite order;

$\left(\mathrm{b}_{2}\right) \operatorname{Norm}_{K / K^{g}}(a)$ is a root of unity in $K^{g}$.

Proof. (a) First, check that the set of all products mult $(a) g$, where $a \in K^{*}, g \in \operatorname{Aut}(K)$, is a subgroup of $\mathrm{GL}_{\mathbb{Q}}(K)$. Let $a_{1}, a_{2} \in K^{*}$ and $g_{1}, g_{2} \in \operatorname{Aut}(K)$. Then, for each $v \in K$,

$$
\operatorname{mult}\left(a_{1}\right) g_{1} \operatorname{mult}\left(a_{2}\right) g_{2}(v)=a_{1}\left(g_{1}\left(a_{2} g_{2}(v)\right)\right)=a_{1}\left(g_{1}\left(a_{2}\right)\right)\left(g_{1}\left(g_{2}(v)\right)\right) .
$$

This yields

$$
\operatorname{mult}\left(a_{1}\right) g_{1} \operatorname{mult}\left(a_{2}\right) g_{2}=\operatorname{mult}\left(a_{1}\left(g_{1}\left(a_{2}\right)\right)\right) g_{1} g_{2} .
$$

From (9) we infer that the inverse of mult $(a) g$ is $\operatorname{mult}\left(g^{-1}\left(a^{-1}\right)\right) g^{-1}$. Thus the set of all products mult $(a) g$ is a subgroup of $\mathrm{GL}_{\mathbb{Q}}(K)$.

On the other hand,

$$
\operatorname{mult}(a) g(1)=\operatorname{mult}(a)(g(1))=\operatorname{mult}(a)(1)=a,
$$

hence the linear operator mult $(a) g$ uniquely determines $a$, and therefore, $g$ as well. This implies that the map

$$
\psi: \mathcal{L}(K) \rightarrow \operatorname{Aut}(K), \quad \operatorname{mult}(a) g \mapsto g,
$$

is well defined. By (9), the map (10) is a group epimorphism and

$$
\operatorname{ker}(\psi)=\operatorname{mult}\left(K^{*}\right) .
$$


Finally, (8) straightforwardly follows from (9).

(b) Let $m:=\operatorname{ord}(g)$. Then it follows from (9) that

$$
(\operatorname{mult}(a) g)^{m}=\operatorname{mult}\left(\prod_{d=0}^{m-1} g^{d}(a)\right) g^{m}=\operatorname{mult}\left(\prod_{d=0}^{m-1} g^{d}(a)\right) .
$$

In turn, (12) implies that $\left(b_{1}\right)$ holds if and only if the right-hand side of (12) is a root of unity. But this right-hand side is $\operatorname{Norm}_{K / K^{g}}(a)$.

Remark 1. The examples below show that, given $a \in K^{*}, g \in \operatorname{Aut}(K)$, the element $\operatorname{mult}(a) g \in \mathcal{L}(K)$ may have a finite order while $a$ is not a root of unity (i.e., its order is infinite).

Examples. 1. Let $K=\mathbb{Q}(\sqrt{2})$. Then $|\operatorname{Aut}(K)|=2$. Let $g \in \operatorname{Aut}(K)$, $g \neq \mathrm{id}_{K}$, and $a=1+\sqrt{2}$. Then $\operatorname{ord}(\operatorname{mult}(a) g)=4, \operatorname{ord}(a)=\infty$.

2. Let $K=\mathbb{Q}(\sqrt{-1})$. Then $|\operatorname{Aut}(K)|=2$. Let $g \in \operatorname{Aut}(K), g \neq \mathrm{id}_{K}$, and $a=(3+4 \sqrt{-1}) / 5$. Then $\operatorname{ord}(\operatorname{mult}(a) g)=2, \operatorname{ord}(a)=\infty$.

Lemma 2. For any finite subgroup $G$ of $\mathcal{L}(K)$, there is a (cyclic) subgroup $H$ of $\mu_{K}$ such that $\operatorname{mult}(H) \subseteq G$ and

(i) the sequence $1 \rightarrow H \stackrel{\text { mult }}{\rightarrow} G \stackrel{\psi}{\rightarrow} \psi(G) \rightarrow 1$ is exact;

(ii) $|G|=|H| \cdot|\psi(G)|$;

(iii) $|H|$ divides $\left|\mu_{K}\right|$;

(iv) $\varphi(|H|)$ divides $n$;

(v) $|\psi(G)|$ divides $\mid$ Aut $(K) \mid$, which divides $n$;

(vi) if $p \geqslant 2$ is a prime integer, then $\nu_{p}(|G|) \leqslant 2 \nu_{p}(n)+1$;

(vii) $|G|$ divides $n \exp (G)$.

Proof. Since $\mu_{K}$ is the set of all elements of finite order in $K^{*}$, and (11) is a group monomorphism, the existence of $H$ and (i) follow from (11). Since $\mu_{K}$ is cyclic, $H$ is cyclic as well.

Statements (ii), (iii), (v) are clear.

Let $\theta$ be a generator of the cyclic group $H$. Then $[\mathbb{Q}(\theta): \mathbb{Q}]=$ $\varphi(\operatorname{ord}(\theta))=\varphi(|H|)$. Whence (iv), because $\mathbb{Q}(\theta)$ is a subfield of $K$.

Since, in the above notation, $\operatorname{ord}(\theta)=|H|$, the definition of $\exp (G)$ implies that $|H|$ divides $\exp (G)$. In view of (ii), this and (v) yield (vii).

Let $\nu_{p}\left(\left|\mu_{K}\right|\right)=d$ and let $\zeta \in \mu_{K}$ be a primitive $p^{d}$ th root of unity. From $\mathbb{Q}(\zeta) \subseteq K$ and $[\mathbb{Q}(\zeta): \mathbb{Q}]=\varphi\left(p^{d}\right)=p^{d-1}(p-1)$ we infer that $p^{d-1}(p-1)$ divides $n$. Hence $\nu_{p}(n) \geqslant d-1=\nu_{p}\left(\left|\mu_{K}\right|\right)-1$. This and (ii), (iii), (v) then imply $\nu_{p}(|G|) \leqslant \nu_{p}\left(\left|\mu_{K}\right|\right)+\nu_{p}(n) \leqslant 2 \nu_{p}(n)+1$, which proves (vi).

For further proofs, Table 1 below summarizes some information from [2] about the Weyl groups of irreducible root systems $R$ of type R: 


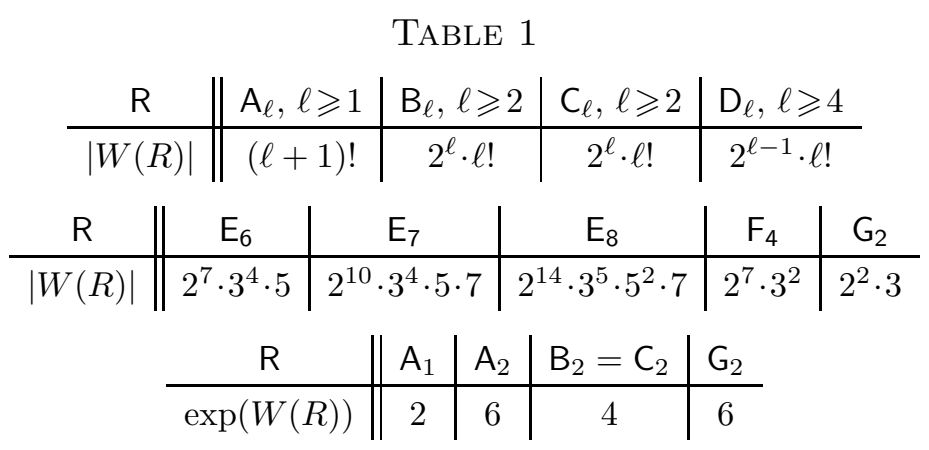

Lemma 3. Let $W(R)$ be the Weyl group of a root system $R$. Then

$$
\nu_{2}(|W(R)|) \geqslant[(\operatorname{rk}(R)+1) / 2] .
$$

Proof. First, by Legendre's formula (see, e.g., [4, Thm. 2.6.1 ]), we have $\nu_{2}(m !)=\sum_{k=1}^{\infty}\left[m / 2^{k}\right] \geqslant[m / 2]+[m / 4]$, which readily yields

$$
\nu_{2}(m !) \geqslant[(m+1) / 2] \quad \text { for } m \neq 1,3 \text {. }
$$

Next, suppose that $R$ is irreducible of type R. Then (13) directly follows from Table 1 and (14).

In the case of an arbitrary root system

$$
R=R_{1}+\cdots+R_{d},
$$

where $R_{i}$ is an irreducible root system for every $i$, we have

$$
\operatorname{rk}(R)=\operatorname{rk}\left(R_{1}\right)+\cdots+\operatorname{rk}\left(R_{d}\right)
$$

and $W(R)$ splits into the product

$$
W(R)=W\left(R_{1}\right) \times \cdots \times W\left(R_{d}\right)
$$

where $W\left(R_{i}\right)$ is the Weyl group of $R_{i}$. It follows from (17) that, for every prime integer $p \geqslant 2$,

$$
\nu_{p}(|W(R)|)=\sum_{i=1}^{d} \nu_{p}\left(\left|W\left(R_{i}\right)\right|\right) .
$$

Given that for every $W\left(R_{i}\right)$ the desired inequality is proved, we then deduce from (18) that

$$
\nu_{2}(|W(R)|) \geqslant \sum_{i=1}^{d}\left[\left(\operatorname{rk}\left(R_{i}\right)+1\right) / 2\right] .
$$

Now (13) follows from (19) because of the inequality

$$
[(a+b+1) / 2] \leqslant[(a+1) / 2]+[(b+1) / 2],
$$

which holds for all integers $a$ and $b$. To prove (20), note that if we replace $a$ by $a+2$, then the both sides of (20) would increase by 1 . So 
validity of (20) for any $a$ and $b$ follows from its evident validity for $a=0$ and $a=1$.

Below some of the arguments are based on the information that readily follows from Table 1 . It is convenient to collect it in Table 2, where we use the same notation as in Table 1 and, for every prime integer $p \geqslant 2$, put $\nu_{p}(\mathrm{R}):=\nu_{p}(|W(R)|)$.

TABLE 2

\begin{tabular}{c||c|c|c|c|c|c|c|c|c|c|c|c|c|c|c|c}
\multicolumn{10}{c}{$\mathrm{R}=\mathrm{A}_{\ell}$} \\
$\qquad$ & 1 & 2 & 3 & 4 & 5 & 6 & 7 & 8 & 9 & 10 & 11 & 12 & 13 & 14 & 15 & 16 \\
\hline$\nu_{2}(\mathrm{R})$ & 1 & 1 & 3 & 3 & 4 & 4 & 7 & 7 & 8 & 8 & 10 & 10 & 11 & 11 & 15 & 15 \\
\hline$\nu_{3}(\mathrm{R})$ & 0 & 1 & 1 & 1 & 2 & 2 & 2 & 4 & 4 & 4 & 5 & 5 & 5 & 6 & 6 & 6
\end{tabular}

\begin{tabular}{c||c|c|c|c|c|c|c|c|c|c|c|c|c|c|c}
\multicolumn{10}{c}{$\mathrm{R}=\mathrm{B}_{\ell}$ and $\mathrm{C}_{\ell}$} \\
$\qquad$ & 2 & 3 & 4 & 5 & 6 & 7 & 8 & 9 & 10 & 11 & 12 & 13 & 14 & 15 & 16 \\
\hline$\nu_{2}(\mathrm{R})$ & 3 & 4 & 7 & 8 & 10 & 11 & 15 & 16 & 18 & 19 & 22 & 23 & 25 & 26 & 31 \\
\hline$\nu_{3}(\mathrm{R})$ & 0 & 1 & 1 & 1 & 2 & 2 & 2 & 4 & 4 & 4 & 5 & 5 & 5 & 6 & 6
\end{tabular}

\begin{tabular}{c||c|c|c|c|c|c|c|c|c|c|c|c|c}
\multicolumn{10}{c}{$\mathrm{R}=\mathrm{D}_{\ell}$} \\
$\ell$ & 4 & 5 & 6 & 7 & 8 & 9 & 10 & 11 & 12 & 13 & 14 & 15 & 16 \\
\hline$\nu_{2}(\mathrm{R})$ & 6 & 7 & 9 & 10 & 14 & 15 & 17 & 18 & 21 & 22 & 24 & 25 & 30 \\
\hline$\nu_{3}(\mathrm{R})$ & 1 & 1 & 2 & 2 & 2 & 4 & 4 & 4 & 5 & 5 & 5 & 6 & 6
\end{tabular}

\begin{tabular}{c||c|c|c|c|c}
$\mathrm{R}$ & $\mathrm{E}_{6}$ & $\mathrm{E}_{7}$ & $\mathrm{E}_{8}$ & $\mathrm{~F}_{4}$ & $\mathrm{G}_{2}$ \\
\hline$\nu_{2}(\mathrm{R})$ & 7 & 10 & 14 & 7 & 2 \\
\hline$\nu_{3}(\mathrm{R})$ & 4 & 4 & 5 & 2 & 1
\end{tabular}

Below, for every type $\mathrm{R}$ of root systems, we put $\varnothing+\mathrm{R}:=\mathrm{R}, 0 \mathrm{R}=\varnothing$, and, by definition, $\operatorname{rk}(\varnothing)=\nu_{p}(\varnothing)=0$ for any $p$.

Proposition 2. Let $R$ be a reduced root system of type $\mathrm{R}$.

(i) If $\mathrm{R}=\mathrm{S}_{1}+\mathrm{S}_{2}$, then

$$
\nu_{2}\left(\mathrm{~S}_{1}\right) \leqslant \nu_{2}(\mathrm{R})-\left[\left(\operatorname{rk}\left(\mathrm{S}_{2}\right)+1\right) / 2\right]
$$

In particular, if $\mathrm{R}_{i}$ is the type of $R_{i}$ in (15), then

$$
\nu_{2}\left(\mathrm{R}_{i}\right) \leqslant \nu_{2}(\mathrm{R})-\left[\left(n-\operatorname{rk}\left(\mathrm{R}_{i}\right)+1\right) / 2\right]<\nu_{2}(\mathrm{R}) .
$$


(ii) If $\nu_{2}(\mathrm{R}) \leqslant 3$, then

$$
\begin{gathered}
\mathrm{R}=a_{1} \mathrm{~A}_{1} \dot{+} a_{2} \mathrm{~A}_{2} \dot{+} a_{3} \mathrm{~A}_{3} \dot{+} a_{4} \mathrm{~A}_{4} \dot{+} b_{2} \mathrm{~B}_{2} \dot{+} g_{2} \mathrm{G}_{2}, \\
a_{1}+2 a_{2}+3 a_{3}+4 a_{4}+2 b_{2}+2 g_{2}=\mathrm{rk}(\mathrm{R}), \\
a_{1}+a_{2}+3 a_{3}+3 a_{4}+3 b_{2}+2 g_{2}=\nu_{2}(\mathrm{R}), \\
a_{2}+a_{3}+a_{4}+g_{2}=\nu_{3}(\mathrm{R}) .
\end{gathered}
$$

(iii) If $\nu_{3}(\mathrm{R}) \leqslant 1$, then

$$
\begin{gathered}
\mathrm{R}=\mathrm{X} \dot{+} a_{1} \mathrm{~A}_{1} \dot{+} b_{2} \mathrm{~B}_{2}, \text { where } \\
\mathrm{X} \in\left\{\mathrm{A}_{2}, \mathrm{~A}_{3}, \mathrm{~A}_{4}, \mathrm{~B}_{3}, \mathrm{~B}_{4}, \mathrm{~B}_{5}, \mathrm{C}_{3}, \mathrm{C}_{4}, \mathrm{C}_{5}, \mathrm{D}_{4}, \mathrm{D}_{5}, \mathrm{G}_{2}, \varnothing\right\}, \\
\operatorname{rk}(\mathrm{X})+a_{1}+2 b_{2}=\mathrm{rk}(\mathrm{R}), \\
\nu_{2}(\mathrm{X})+a_{1}+3 b_{2}=\nu_{2}(\mathrm{R}) .
\end{gathered}
$$

In this case, $W(R)$ contains a subgroup isomorphic to the Weyl group of a root system of type $\mathrm{mA}_{1}$, where

$$
m= \begin{cases}\operatorname{rk}(\mathrm{R}) & \text { if } \mathrm{X}=\mathrm{B}_{3}, \mathrm{~B}_{4}, \mathrm{~B}_{5}, \mathrm{C}_{3}, \mathrm{C}_{4}, \mathrm{C}_{5}, \mathrm{D}_{4}, \mathrm{G}_{2}, \varnothing, \\ \mathrm{rk}(\mathrm{R})-1 & \text { if } \mathrm{X}=\mathrm{A}_{2}, \mathrm{~A}_{3}, \mathrm{D}_{5}, \\ \mathrm{rk}(\mathrm{R})-2 & \text { if } \mathrm{X}=\mathrm{A}_{4} .\end{cases}
$$

Proof. Combining Lemma 3, (16), (18), and Table 2 yields the proof of all but the last claim of (iii). To prove the latter, we note that if $Y$ is a root system of type $Y$, then, for $Y=A_{1}, B_{\ell}, C_{\ell}, D_{\ell}(\ell$ even), and $\mathrm{G}_{2}$, there is a subset of $\operatorname{rk}(\mathrm{Y})$ strongly orthogonal (hence orthogonal) roots in $Y$ (see [1, Thms. 3.1, 5.1]; cf. 2, Chap. VI, $\S 1$, Sect. 3, Cor. of Thm. 1, Exer. 15]). The subgroup of $W(Y)$ generated by reflections corresponding to these roots is isomorphic to the Weyl group of a root system of type $\operatorname{rk}(Y) A_{1}$. This proves the claim in the case $X=B_{3}, B_{4}$, $B_{5}, C_{3}, C_{4}, C_{5}, D_{4}, G_{2}, \varnothing$. The root systems of types $A_{2}, A_{3}, A_{4}$, and $D_{5}$ clearly contain closed subsystems of respectively types $A_{1}, A_{1}+A_{1}$, $A_{1}+A_{1}$, and $D_{4}$. This implies the claim in the remaining two cases.

Proposition 3. Let $K$ be a number field of degree $n$ over $\mathbb{Q}$ and let $m$ be a positive integer. If the group $\mathcal{L}(K)$ contains a subgroup $G$ isomorphic to the Weyl group of a root system of type $m \mathrm{~A}_{1}$, then the following hold:
(a) $2^{m-1}$ divides $n$;
(b) if $m=n$, then $n=1$ or 2 ;
(c) if $m=n-1$, then $n=2$ or 4 ;
(d) if $m=n-2$, then $n=3$ or 4 .

Proof. Table 1 and (17) yield $\exp (G)=2$ and $|G|=2^{m}$. Hence, by Lemma 2(vii), $2^{m}$ divides $2 n$, whence (a). In turn, (a) implies (b), (c), and $(\mathrm{d})$. 
Proposition 4. Let $K$ be a number field of degree $n$ over $\mathbb{Q}$. If the group $\mathcal{L}(K)$ contains a finite subgroup $G$ isomorphic to the Weyl group $W(R)$ of a root system $R$ of type $\mathrm{R}$ and rank $n$, then $n \in\{1,2,4\}$.

Proof. First, in Step 1, we shall show that $n \in\{1,2,4,6,8,16\}$. Then, in Steps 2, 3, and 4, we shall consider respectively the cases $n=6,8$, and 16, and eliminate each of them.

Step 1.

Lemma 2(vi) and (13) yield

$$
[(n+1) / 2] \leqslant \nu_{2}(|G|) \leqslant 2 \nu_{2}(n)+1 .
$$

Let $n \geqslant 3$. Then $2 \leqslant[(n+1) / 2]$. In view of (23) , this implies that $\nu_{2}(n) \geqslant 1$, i.e., $n$ is even. Since $n / 2 \leqslant[(n+1) / 2]$, from (23) we infer

$$
2^{n / 2} \leqslant 2^{[(n+1) / 2]} \leqslant 2^{2 \nu_{2}(n)+1} \leqslant 2 n^{2} .
$$

In addition, if $n$ is not a power of 2 , then $2^{\nu_{2}(n)} \cdot 3 \leqslant n$, so (24) yields

$$
2^{n / 2} \leqslant 2^{2 \nu_{2}(n)+1} \leqslant 2(n / 3)^{2} .
$$

If in (25) we replace $n$ by $n+2$ then the left-hand side will be multiplied by 2 while the right-hand side will be multiplied by $(1+$ $2 / n)^{2}<2$, because $n>4$. Taking into account that (25) becomes equality if $n=6$, we conclude that $n=6$ if $n \geqslant 3$ is not a power of 2 .

Now suppose that $n=2^{s}$, where $s \geqslant 2$. Then (23) yields

$$
2^{s-1} \leqslant 2 s+1
$$

and therefore $s=2,3$ or 4 , i.e., $n=4,8$ or 16 respectively.

Taking into account all $n<3$, we conclude that $n \in\{1,2,4,6,8,16\}$.

In Steps 2, 3, and 4, we use the notation of (15), (17) introduced in the proof of Lemma 3 . The type of $R_{i}$ is denoted by $\mathrm{R}_{i}$.

Step 2 .

Arguing on the contrary, assume that $n=6$. Then (23) yields $3 \leqslant$ $\nu_{2}(|G|) \leqslant 3$, i.e., $\nu_{2}(|G|)=\nu_{2}(\mathrm{R})=3$. From this and Proposition $2($ ii) we deduce that $\mathrm{R}=a_{1} \mathrm{~A}_{1}+a_{2} \mathrm{~A}_{2}$, where

$$
a_{1}+2 a_{2}=6, \quad a_{1}+a_{2}=3 .
$$

Hence $a_{1}=0, a_{2}=3$, i.e., $\mathrm{R}=3 \mathrm{~A}_{2}$. This and Table 1 yield

$$
|G|=6^{3}, \exp (G)=6 .
$$

On the other hand, $|G|$ divides $6 \exp (G)$ by Lemma 1(vii). This contradicts (26). Hence $n \neq 6$.

\section{Step 3.}

Arguing on the contrary, assume that $n=8$. Then Lemma 2(vi) yields $\nu_{3}(\mathrm{R}) \leqslant 1$. Hence by Proposition $2($ iii), the group $G$ contains 
a subgroup isomorphic to the Weyl group of a root system of type $(8-2) \mathrm{A}_{1}$. This contradicts Proposition $3(\mathrm{~d})$, so we conclude that $n \neq 8$.

Step 4

Arguing on the contrary, assume that $n=16$. Then Lemma 2(vi) yields $\nu_{3}(\mathrm{R}) \leqslant 1$. Therefore, by Proposition $2($ iii), the group $G$ contains a subgroup isomorphic to the Weyl group of a root system of type $(16-2) \mathrm{A}_{1}$. This contradicts Proposition $3(\mathrm{~d})$. Hence $n \neq 16$.

\section{Proofs of Theorems 1 and 2}

Proof of Theorem 1.

(i) $\Rightarrow$ (ii) Assume that (i) holds. In view of Proposition 4, we have to show that if $n=4$, then $\mathrm{R}$ is either $\mathrm{A}_{2}+\mathrm{B}_{2}$ or $2 \mathrm{~A}_{1}+\mathrm{A}_{2}$.

So, let $n=4$. Then Lemma 2(v) (whose notation we use) yields

$$
|\psi(G)|=1,2 \text {, or } 4 \text {. }
$$

Next, we have $\nu_{2}(n)=2, \nu_{3}(n)=0$. Therefore, Lemma 2(vi) yields $\nu_{2}(\mathrm{R}) \leqslant 5, \nu_{3}(\mathrm{R}) \leqslant 1$. Proposition 2 (iii) and Table 2 then infer that $\mathrm{R}$ is one of the following root systems

$$
\begin{gathered}
A_{4}, A_{1}+A_{3}, A_{1}+B_{3}, A_{1}+C_{3}, 2 A_{1}+A_{2}, \\
2 A_{1}+B_{2}, 4 A_{1}, A_{2}+B_{2}, 2 A_{1}+G_{2}, B_{2}+G_{2}
\end{gathered}
$$

Assume that $\mathrm{R}=\mathrm{A}_{4}$. Then $G$ is isomorphic to $\mathrm{Sym}_{5}$. Since the only proper normal subgroup of $\mathrm{Sym}_{5}$ is $\mathrm{Alt}_{5}$ (see, e.g., [5, Thm. 4.7]), and $\mathrm{Alt}_{5}$ is noncyclic, Lemma 2 implies that $H$ is trivial, whence $|G|=5$ ! divides $n=4$. This contradiction shows that, in fact, $\mathrm{R} \neq \mathrm{A}_{4}$.

Assume that $\mathrm{R}$ is one of the root systems

$$
A_{1}+B_{3}, A_{1}+C_{3}, 2 A_{1}+B_{2}, 4 A_{1}, 2 A_{1}+G_{2}, B_{2}+G_{2} .
$$

Then, by Proposition 2(iii), the group $G$ contains a subgroup isomorphic to the Weyl group of a root system of type $4 \mathrm{~A}_{1}$. This contradicts Proposition 3(b). Hence, in fact, $\mathrm{R}$ is none of the root systems (28).

Finally, assume that $\mathrm{R}=\mathrm{A}_{1}+\mathrm{A}_{3}$. Then $G$ is isomorphic to $\mathrm{Sym}_{2} \times$ $\mathrm{Sym}_{4}$ and

$$
|G|=2^{4} \cdot 3 .
$$

Lemma 2(ii) and (29) imply that, respectively to (27), we have $|H|=$ $2^{4} \cdot 3,2^{3} \cdot 3$, or $2^{2} \cdot 3$, and, accordingly, $\varphi(|H|)=2^{4}, 2^{3}$, or $2^{2}$. Since only the last integer divides 4 , by Lemma 2 (iv) we conclude that $|\psi(G)|=$ 4. Hence $G$ contains a cyclic subgroup mult $(H)$, whose generator has order 12. But the maximum of orders of elements of $\mathrm{Sym}_{2} \times \mathrm{Sym}_{4}$ is 6 (see, e.g., [5, p. 6]). This contradiction shows that, in fact, $R \neq A_{1}+A_{3}$.

The proof of (i) $\Rightarrow$ (ii) is now completed. 
(ii) $\Rightarrow$ (i) If $R \in\left\{A_{1}, A_{2}, B_{2}, G_{2}, 2 A_{1}\right\}$, then (i) follows from Proposition 1 and Definition 1 .

Consider the case $\mathrm{R}=\mathrm{A}_{2}+\mathrm{B}_{2}$.

Let $K$ be the biquadratic field $\mathbb{Q}(\sqrt{-3}, \sqrt{-1})$. Then

$$
K=\mathbb{Q}(\sqrt{-3}) \otimes_{\mathbb{Q}} \mathbb{Q}(\sqrt{-1}) .
$$

This equality determines the natural homomorphism

$$
\mathcal{L}(\mathbb{Q}(\sqrt{-3})) \times \mathcal{L}(\mathbb{Q}(\sqrt{-1})) \rightarrow \mathcal{L}(K),
$$

whose restriction to $\operatorname{Aut}(\mathbb{Q}(\sqrt{-3})) \times \operatorname{Aut}(\mathbb{Q}(\sqrt{-1}))$ is an isomorphism with $\operatorname{Aut}(K)$ (see [3, Chap. VIII, $\S 1$, Thm. 5]). The kernel of homomorphism (30) is $\left\{\left(\operatorname{mult}(a), \operatorname{mult}\left(a^{-1}\right)\right) \mid a \in \mathbb{Q}^{*}\right\}$.

Let $R_{1}$ and $R_{2}$ be respectively the realizations of type $\mathrm{A}_{2}$ in $\mathbb{Q}(\sqrt{-3})$ and of type $B_{2}$ in $\mathbb{Q}(\sqrt{-1})$ constructed in the proof of Proposition 1. Since $-1 \notin W\left(R_{1}\right)$, the restriction of homomorphism (30) to the subgroup $W\left(R_{1}\right) \times W\left(R_{2}\right)$ is an embedding. Therefore, its image is the subgroup of $\mathcal{L}(K)$ isomorphic to the Weyl group of a root system of type $A_{2}+B_{2}$. This proves that (i) holds if $R=A_{2}+B_{2}$.

Now consider the case $\mathrm{R}=\mathrm{A}_{2}+2 \mathrm{~A}_{1}$.

If $R_{3}$ is a subset of $R_{2}$, which is a realization of type $2 \mathrm{~A}_{1}$ in $K$, then the restriction of homomorphism (30) to $W\left(R_{1}\right) \times W\left(R_{3}\right)$ is the subgroup of $\mathcal{L}(K)$ isomorphic to the Weyl group of a root system of type $A_{2}+2 A_{1}$. Thus (i) holds if $R$ is of this type.

This completes the proof of (ii) $\Rightarrow$ (i) and that of Theorem 1 .

Proof of Theorem 2. (i) $\Rightarrow$ (ii) In view of Theorem 1 and Definition 1 , we have to show that if $R=A_{2}+B_{2}$ or $A_{2}+2 A_{1}$, then $R$ admits no realizations in the number fields. Arguing on the contrary, assume that this is not the case, i.e., $\mathrm{R}$ admits a realization in a number field $K$.

The linear space $K$ over $\mathbb{Q}$ is then a direct sum of two 2-dimensional linear subspaces $L_{1}$ and $L_{2}$ such that

(a) $L_{i}$ is the linear span of $R_{i}:=R \bigcap L_{i}$ over $\mathbb{Q}$ for every $i$;

(b) $R_{1}$ is a root system in $L_{1}$ of type $\mathrm{A}_{2}$;

(c) $R_{2}$ is a root system in $L_{2}$ of type $\mathrm{B}_{2}$ or $2 \mathrm{~A}_{1}$;

(d) $R=R_{1}+R_{2}$.

Let $\iota$ : $\mathrm{GL}_{\mathbb{Q}}\left(L_{1}\right) \times \mathrm{GL}_{\mathbb{Q}}\left(L_{2}\right) \hookrightarrow \mathrm{GL}_{\mathbb{Q}}(K)$ be the natural group embedding. Then

$$
W(R)=\iota\left(W\left(R_{1}\right)\right) \times \iota\left(W\left(R_{2}\right)\right) .
$$

In view of (b), the group $\iota\left(W\left(R_{1}\right)\right)$ is isomorphic to $\mathrm{Sym}_{3}$, hence contains an element $z$ of order 3. By (31), we have

$$
L_{2} \subseteq K^{z} .
$$


According to Lemma 1, there are uniquely defined elements $a \in K^{*}$ and $g \in \operatorname{Aut}(K)$ such that $z=\operatorname{mult}(a) g$. From (10) we infer that $\operatorname{ord}(g)$ divides $\operatorname{ord}(z)=3$. Since $\operatorname{ord}(g)$ divides $|\operatorname{Aut}(K)|$, which, in turn, divides $\operatorname{dim}_{\mathbb{Q}}(K)=4$, we conclude that

$$
z=\operatorname{mult}(a) \text {. }
$$

As ord $(z) \neq 1$, we have $a \neq 1$. From this, (33), and (2) we then conclude that $K^{z}=0$ contrary to (32). This completes the proof of (i) $\Rightarrow$ (ii).

(ii) $\Rightarrow$ (i) This follows from Proposition 1.

\section{REFERENCES}

[1] Y. Agaoka, E. Kaneda, Strongly orthogonal subsets in root systems, Hokkaido Math. J. 31 (2002), 107-136.

[2] N. Bourbaki, Groupes et Algébres de Lie, Chaps. IV-VI, Hermann, Paris, 1968.

[3] S. Lang, Algebra, Addison-Wesley, Mass., 1965.

[4] V. H. Moll, Numbers and Functions, Student Math. Library, Vol. 65, AMS, Providence, RI, 2012.

[5] D. Passman, Permutation Groups, Benjamin, New York, 1968.

[6] J.-P. Serre, Complex Semisimple Lie Algebras, Berlin, Springer, 2001. 